\title{
EFFECTS OF ORAL ALFACALCIDOL ON MATURATION OF DENDRITIC CELLS IN GRAVES' DISEASE PATIENTS: A DOUBLE-BLINDED RANDOMIZED CLINICAL TRIAL
}

\section{DYAH PURNAMASARI ${ }^{*}$, SAMSURIDJAL DJAUZI ${ }^{2}$, SITI SETIATI ${ }^{3}$, ALIDA HARAHAP ${ }^{4}$, TJOKORDA G PEMAYUN ${ }^{5}$, JOEDO PRIHARTONO ${ }^{6}$, PRADANA SOEWONDO ${ }^{1}$}

${ }^{1}$ Department of Medicine, Division of Endocrinology, Faculty of Medicine Universitas Indonesia, Indonesia. ${ }^{2}$ Department of Medicine, Division of Allergy Immunology, Faculty of Medicine Universitas Indonesia, Indonesia. ${ }^{3}$ Department of Medicine, Division of Geriatrics, Faculty of Medicine Universitas Indonesia, Indonesia. ${ }^{4}$ Departement of Clinical Pathology, Division of Immunology, Faculty of Medicine

Universitas Indonesia, Indonesia. ${ }^{5}$ Department of Medicine, Division of Endocrinology, Faculty of Medicine Universitas Diponegoro, Indonesia. ${ }^{6}$ Departement of Community Medicine, Faculty of Medicine Universitas Indonesia, Indonesia. Email: dyah_p_irawan@yahoo.com

Received: 10 November 2016, Revised and Accepted: 09 March 2017

ABSTRACT

Objective: Maturity level of dendritic cells (DC) plays a pivotal role in initiating and regulating autoimmunity. In Graves' disease (GD), DCs have more vigorous immune responses than those in healthy controls. Our previous study demonstrated immunoregulatory effects of in vitro 1,25-D3 on the maturation of DC in GD patients. This study aims to evaluate the effect of oral alfacalcidol (1 $\alpha$-D3) on DC maturation in GD patients.

Methods: 25 GD patients with thyrotoxicosis were divided into 2 groups: 12 GD patients receiving oral $1 \alpha$-D3 and 13 GD patients receiving placebo, in addition to the treatment of propylthiouracil. Comparison of DC maturation was performed before and after the oral $1 \alpha$-D3. DC maturation was evaluated based on the expression of DC markers (HLA-DR, CD80, CD40, CD83, CD14, and CD206) and the ratio of cytokines interleukin-12 (IL-12)/IL-10.

Results: After 8 weeks, 8 out of 12 GD patients in the treatment group and 6 out of 13 GD patients in the placebo group still had high fT4 level. The expression of CD80 decreased ( $p=0.48$ ) and CD206 increased ( $p=0.47)$ insignificantly among treatment group. The IL- $12 / \mathrm{IL}-10$ ratio decreased along with the improvement of fT4 level in both groups. No difference in the IL-12/IL-10 ratio between treatment and placebo group.

Conclusion: The effects of oral $1 \alpha-\mathrm{D} 3$ on DC maturation of GD patients have not been clearly demonstrated in this study yet.

Keywords: Vitamin D, Graves' disease, Dendritic cells.

(c) 2017 The Authors. Published by Innovare Academic Sciences Pvt Ltd. This is an open access article under the CC BY license (http://creativecommons. org/licenses/by/4. 0/) DOI: http://dx.doi.org/10.22159/ajpcr.2017.v10i6.16110

\section{INTRODUCTION}

Previously, Graves' disease (GD) had been considered as organ-specific autoimmune disease until an animal study showed that mice with total thyroidectomy still had a high level of cytokine interleukin-12 (IL-12) from its spleen [1]. IL-12, inflammatory cytokine, primarily comes from dendritic cells (DC). In autoimmune disease, maturation of DC gives a potential contribution to the development and maintenance of immune response [2].

Mature DCs have a strong positive expression on major histocompatibility complex (MHC), costimulatory, and DC maturation molecules (HLA-DR, CD80, CD40, and CD83), but have low markers for monocytes (CD14) and mannose receptor (MR/CD206). In contrast, immature DCs have a weak positive expression on $\mathrm{MHC}$, costimulatory, and DC maturation molecules, but they have strong markers for monocytes and MR. Beside IL-12, DC also produce low level of IL-10 which has anti-inflammatory properties. The balance of IL-12 and IL-10 contributes to the activation of naive T lymphocyte cells [2].

DC maturation is influenced by genetic and environmental factors. The genetic factors affecting DC maturation are HLA gene [3] and inflammatory cytokines genes [4,5], while the environmental factors affecting DC maturation are infection [6], thyroid hormone [7], hormonal changes in women related to menstruation approaches, the use of sex-steroid hormone drug [8], immunosuppressants [9], and vitamin D [10]. The previous studies among systemic lupus erythematosus (SLE), multiple sclerosis (MS), and Crohn's disease showed that in vitro 1,25 dihydroxy vitamin D (1,25-D3) impaired the maturation of DC in monocyte-derived DC (MDDC) cultures [10-12].
Although the effect of 1,25-D3 on immunology process was clearly proved in in vitro study, the advantage of vitamin D supplementation among SLE, MS, and Crohn's disease is still on debate [13-16].

In GD, previous studies have showed a relationship between vitamin D deficiency, the polymorphisms of the vitamin D receptor, and vitamin D binding protein with the occurrence of GD $[17,18]$. Vitamin D levels have association with remission status [19]. A study by Kawakami-Tani et al. [20] in Japan among new onset GD patients showed that alfacalcidol ( $1 \alpha$-D3) supplementation along with antithyroid drug (ATD) can reduce the level of thyroid hormone which was faster in the treatment group compared to placebo group. Those effects are likely due to direct antithyroid actions of $1 \alpha-\mathrm{D} 3$ on the thyroid gland. However, the immunological process behind the $1 \alpha$-D3 induced-thyroid hormone reduction is not well defined yet.

In the previous study, we already showed that in vitro 1,25-D3 supplementation in MDDC cultures of GD patients will interfere the maturation of DC by decreasing the expression of MHC class II molecule (HLA-DR), costimulatory molecules (CD80 and CD40), and DC marker (CD83) as well as increasing the expression of monocyte (CD14) [21]. The result showed that in vitro 1,25-D3 intervention has a positive effect in the immunology process among GD patients. In this study, we aim to evaluate the effect of in vivo $1 \alpha$-D3 supplementation to DC maturation in GD patients.

\section{METHODS}

\section{Design}

Between May 2014 and March 2015, we found 29 GD patients who were still in hyperthyroid condition at the outpatient clinic of 
Endocrinology Department of Dr. Cipto Mangunkusumo Hospital in Jakarta, Indonesia. The diagnosis of GD was made based on clinical manifestation (tachycardia, fine tremor, excessive sweat, diffuse struma, and ophthalmopathy) and laboratory data (low thyroid stimulating hormone [TSH] level, increased fT4 level, and positive thyroid receptor antibody [TRAb]). Patients who took vitamin D supplementation were pregnant or had comorbidities, i.e., diabetes mellitus (DM), chronic kidney disease, and other autoimmune diseases were excluded from the study. Out of 29 patients, 25 patients completed the study, while 4 patients were unable to complete the study due to laboratory technical problems.

This is a double-blinded, randomized clinical trial (RCT) study. A 4-block randomization was performed in $25 \mathrm{GD}$ patients to categorize them into two groups, i.e., the treatment and placebo group. There were 12 GD patients in the treatment group who received propylthiouracil (PTU) $300 \mathrm{mg}$ daily with supplementation of $1 \alpha-\mathrm{D} 3$ at dose of $1.5 \mathrm{mcg}$ once daily for 8 weeks; while 13 GD patients in the placebo groups received PTU 300 mg daily and placebo tablets for 8 weeks (Fig. 1).

The patients were scheduled to have hospital visits every two weeks, and the responsible physician evaluated the clinical and laboratory data. All patients gave their informed consent. The study received approval from the Ethics Committee of Faculty of Medicine, Universitas Indonesia no. 489/H2.F1/ETIK/2014.

\section{Biological materials and measurements}

After randomization, blood specimens were collected from venous blood at certain times; then underwent centrifugation. The serum was stored at $-80^{\circ} \mathrm{C}$ for laboratory evaluation, and the rests were processed for MDDC cultures. Laboratory evaluation consists of vitamin 25-D3 (Euroimmun) and TRAb (DRG) level before supplementation as well as TSH and fT4 (ROCHE) level before supplementation, at week 4 and week 8 (after supplementation).
MDDC culture: Mo-DC differentiation

MDDC cultures were performed before and after supplementation. The isolation of PBMC and monocytes followed by lipopolysaccharide (LPS) stimulation was done based on the method that has been performed by Budiyati et al. [22], The isolation of PBMC was conducted through density differences using Ficoll-Paque premium (GE Healthcare). The specimens were dissolved in the Roswell Park Memorial Institute (RPMI) medium at cell density of $1 \times 10^{6} / \mathrm{ml}$. Isolation of monocytes was performed using the adherence method. One $\mathrm{ml}$ of DC culture media (DCCM) was added after the monocytes adhered to the base of the plate. DCCM consists of RPMI 1640 medium, 10\% fetal bovine serum (Gibco), $800 \mathrm{U} / \mathrm{ml}$ of Granulocyte-macrophage colony-stimulating factor (BD Bioscience Pharmingen), and $1000 \mathrm{U} / \mathrm{ml}$ of IL-4 (BD Bioscience Pharmingen). At the end of those procedures, the cells then were incubated at $37^{\circ} \mathrm{C}$ with $\mathrm{CO}_{2} 5 \%$ for the next 5 days.

\section{MDDC culture: DC maturation and harvesting}

Additional 500 ng LPS was added (Sigma-Aldrich) to cultures on the fifth day (D5), and the incubation was then continued for further 2 days. After cell harvesting and separation procedures from the supernatant, the cells can be processed for monoclonal antibody staining, while the supernatant was stored at $-20^{\circ} \mathrm{C}$ for evaluating IL- 12 and IL- 10 cytokines.

\section{Flow cytometry analysis}

The flow cytometry analysis was operated using fluorescence-activated cell sorting (FACS) Calibur (BD Biosciences). The fluorochrome-conjugated antibodies (BD Biosciences Pharmingen) used in flow cytometry technique are HLA-DR fluorescein isothiocyanate (FITC) (G46-6, mouse IgG2ak), CD40 phycoerythrin (PE) (5C3, mouse IgG1א), CD80 PE-Cyanine5 (PECy5)

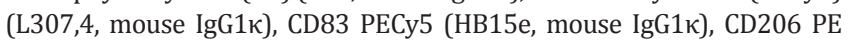
(19,2, mouse IgG1א), and CD14 FITC (M5E2, mouse IgG2ак).

The expression of HLA-DR, CD80, CD40, and CD206 were presented in the form of mean fluorescence intensities, while the expression of CD14

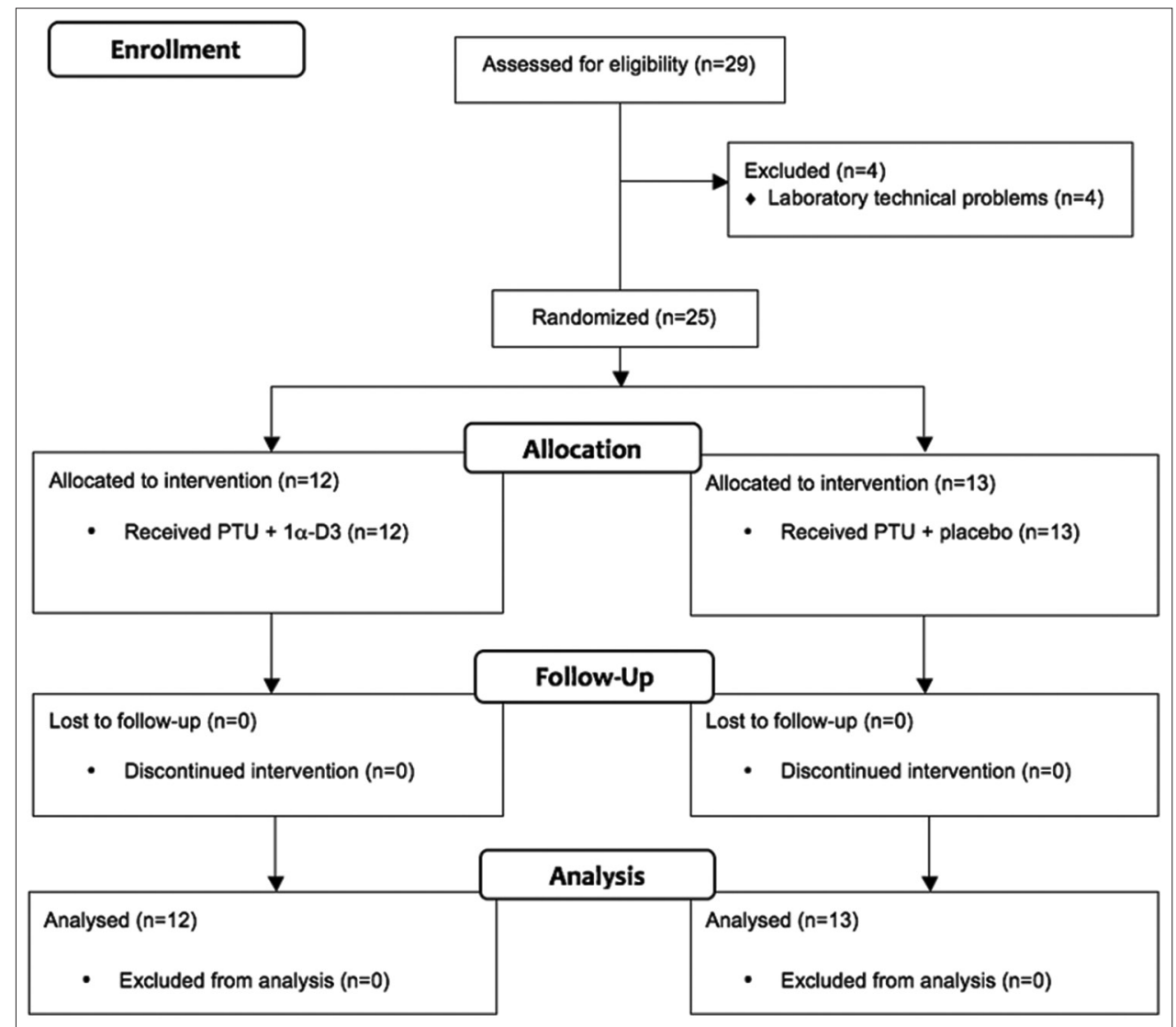

Fig. 1: Flow diagram of the randomization 
and CD83 were presented in numbers of positive cells. Measurement of cytokine levels (IL-12 and IL-10) was done using Human IL-12 p70 (Quantikine HS ELISA Kit - R\&D Systems kit) and Human IL-10 (Quantikine ELISA Kit - R\&D Systems).

\section{Statistical analysis}

Data were analyzed following per-protocol analysis and presented in mean value \pm standard deviation when they have normal distribution and in median value (minimum and maximum value) when they did not have normal distribution. The evaluation of mean or median value for DC marker expression and ratio of IL-12/IL-10 cytokines between before and after supplementation in the treatment and placebo group were carried out using paired t-test when the data had normal distribution and using Wilcoxon test when the data were not normally distributed.

\section{RESULTS}

Subject characteristics can be seen in Table 1 .

Data are presented in mean value \pm SD when they were normally distributed and in median value (minimum and maximum value) when they did not have normal distribution.

Following the randomization, there were two study groups (the treatment and placebo group), which had similar baseline characteristics $(\mathrm{p}>0.05)$.

Effects of in vivo $1 \alpha$-D3 on the expression of DC markers and the ratio of cytokines IL-12/IL-10 in MDDC cultures of GD patients The effects of in vivo $1 \alpha$-D3 supplementation on DC maturation were evaluated by comparing each expression of DC marker in the MDDC culture before and after 8-week supplementation. The expression of CD80 on the $7^{\text {th }}$ day of MDDC culture was lower in the treatment group $(p=0.48)$ after supplementation; while in the placebo group, there was an increase $(p=0.22)$. The expression of CD206 on the $7^{\text {th }}$ day of MDDC culture increased in the treatment group ( $\mathrm{p}=0.47)$; while in the placebo group, it decreased ( $\mathrm{p}=0.18$ ) (Fig. 2). Both CD40 and CD83 expression in both groups increased insignificantly, while the expression of CD14 decreased insignificantly in both groups. Following 8-week supplementation, the ratio of IL-12/IL-10 levels reduced insignificantly in both groups $(\mathrm{p}=0.53$ and $\mathrm{p}=0.08)$.

\section{Effects of oral $1 \alpha$-D3 on fT 4 level in GD patients}

As many 8 out of 12 GD patients in treatment group and 6 out of 13 GD patients in placebo group still had high fT4 level. Both groups had lower thyroid level (fT4) after 8-week supplementation (3.29 2.05 vs. $2.88 \pm 3.01 ; \mathrm{p}=0.34)$. The percentage of lower fT4 level in both groups were not different (58.49 (0.00-89.33 vs. - 76.05-88.16); $\mathrm{p}=0.77)$ (Fig. 3).

\section{DISCUSSION}

DC has a significant role in initiating and maintaining the autoimmune response [2]. DC maturation process is influenced by genetic and environmental factors. One of the environmental factors contributing to DC maturation is vitamin D. As an immunomodulator; vitamin D regulates several innate and adaptive immune responses found in autoimmune diseases, including inhibition of DC maturation [23]. This study reveals further knowledge regarding the immunoregulatory effect of vitamin D, mainly on inhibition of DC maturation in GD patients. This is the first study reporting the effect of oral vitamin $\mathrm{D}$ on inhibition of DC maturation in GD patients.

The mean value of vitamin 25-D3 level in GD patients of our study was $25 \pm 6.62 \mathrm{ng} / \mathrm{ml}$. This value is higher than a value reported in Japan. Yasuda et al. [19] reported the mean value of vitamin 25-D3 level in GD patients was $14.5 \pm 2.9 \mathrm{ng} / \mathrm{ml}$ in non-remission versus $18.2 \pm 5.1 \mathrm{ng} / \mathrm{ml}$ in remission group. About $76 \%$ patients in our study still had vitamin D insufficiency $(<30 \mathrm{ng} / \mathrm{ml})$. This finding is similar to studies on other autoimmune diseases [24].

Effects of in vivo 1 $\alpha$-D3 on the expression of DC markers in MDDC cultures of GD patients

This study design refers to a study conducted by Kawakami-Tani et al. which reported that there is a greater reduction of fT4 level in GD patients receiving methimazole (MMI) and $1 \alpha$-D3 supplementation than those who only receive MMI alone [20]. The present study wants to identify whether the greater reduction of the fT4 level is also accompanied by immunological process, particularly inhibition of DC maturation on molecular aspects.

The results of our study demonstrate that oral supplementation of $1 \alpha$-D3 for 8 weeks has not yet inhibited DC maturation, which is

Table 1: Subject characteristics $(n=25)$

\begin{tabular}{|c|c|c|}
\hline Variable & $\begin{array}{l}\text { Mean } \\
\text { value } \pm S D\end{array}$ & Median (minimum-maximum) \\
\hline Age (years) & $36 \pm 8.81$ & \\
\hline $\begin{array}{l}\text { Duration of } \\
\text { illness (months) }\end{array}$ & & $24(2-26.6)$ \\
\hline $\mathrm{TSH}(\mu \mathrm{IU} / \mathrm{l})$ & 0.01 & \\
\hline fT4 (ng/dl) & & $5.04(2.51-7.77)$ \\
\hline TRAb (U/l) & & $14.174(3.304-87.738)$ \\
\hline Ca ion $(\mathrm{mmol} / \mathrm{l})$ & $1.13 \pm 0.05$ & \\
\hline Vitamin $25-\mathrm{D} 3 \mathrm{ng} / \mathrm{ml}$ & $25 \pm 6.62$ & \\
\hline
\end{tabular}

Data are presented in mean value \pm SD when they were normally distributed and in median value (minimum and maximum value) when they did not have normal distribution

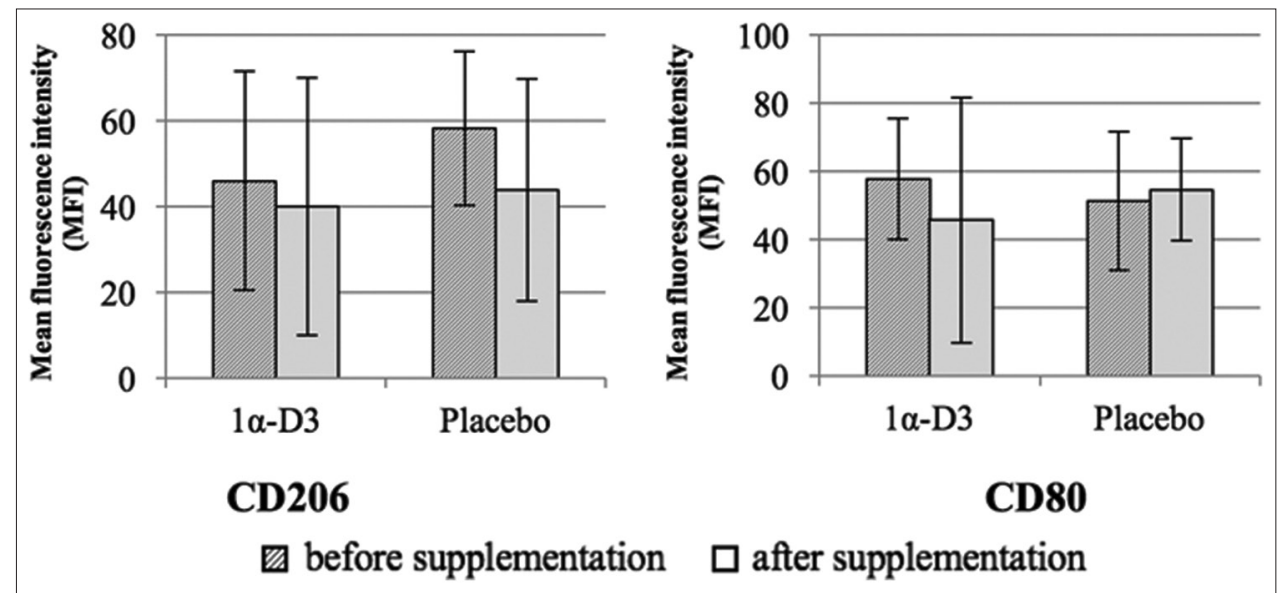

Fig. 2: The expression of CD206 and CD80 in cultures before and after the supplementation of 1 $\alpha$-D3 


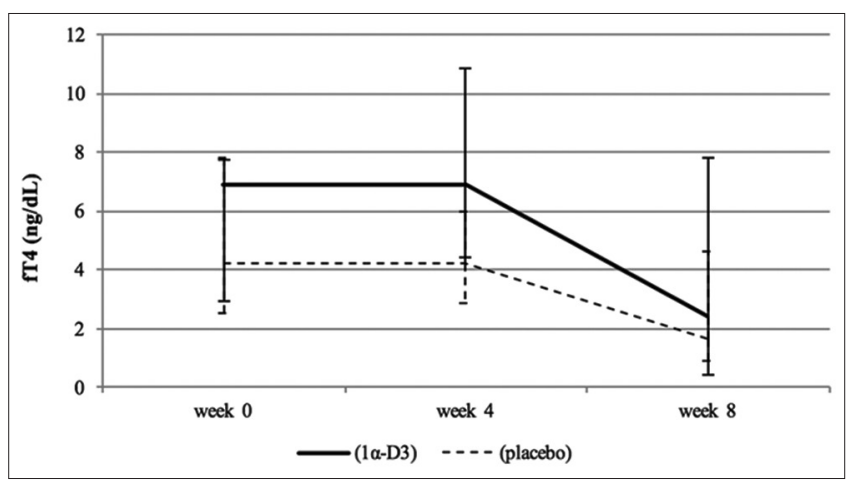

Fig. 3: The level of fT4 before and after 8-week supplementation of $1 \alpha-\mathrm{D} 3$ compared to placebo

characterized by insignificant lower expression of CD80 costimulatory molecule and persistent expression of MR (CD206). Although the results have not been significant yet, oral supplementation of $1 \alpha$-D3 tends to demonstrate inhibition of DC maturation, which is characterized by lower expression of costimulatory molecule and the persistence of MR expression, while placebo supplementation tends to have higher expression of costimulatory molecule and lower MR expression. Lower costimulatory molecule expression and the persistence of MR molecules suggest that there is inhibition of DC maturation. There were no significant differences between oral $1 \alpha$-D3 supplementation and placebo treatment on other DC markers, i.e., CD83, CD40, and CD14.

Our study is the first RCT evaluating the effect of oral vitamin D on immune cells profile (DC maturation) in GD. It is also the first study observing the effect of oral vitamin D supplementation on MR expression in autoimmune disease. A systematic review in 2012 about vitamin D supplementation in autoimmune diseases shows that out of 270 analyzed studies, there are only two RCTs; while others are observational study. Both RCTs reported the effect of $0.5 \mathrm{mcg}$ oral $1 \alpha$-D3 supplementation on lower needs of insulin and improved function of beta cells in patients with DM1 [25]. There is one RCT in 2013, which has not been incorporated into the systematic review. It discussed the effect of oral vitamin D on improved disease activity, inflammatory cytokines, and hemostatic profile of patients with SLE [15]. The previous three RCTs involving a greater number of autoimmune patients did not evaluate the profile of immune cells.

Our study is the second study which evaluates the effect of oral vitamin D on DC maturation in autoimmune disease. The first study is a study of drug-placebo without randomization conducted by Bartels et al. in 19 patients with Crohn's disease. The results of that study showed that there was significant lower expression of costimulatory molecule (CD80) ( $\mathrm{p}=0.04)$ in the 25-D3 group than those in placebo group, but the expressions of HLA and other costimulatory molecule were not different $(p>0.05)[26]$.

In Bartels et al. study, in vivo supplementation only demonstrated partial changes on DC maturation markers [26]. In our study, in vivo vitamin D supplementation reduced the expression of costimulatory molecule (CD80) and maintained the expression of MR (CD206) insignificantly in treatment group. Although the results were not significant, they showed the trend of vitamin D's positive effect since reverse changes occurred in placebo group, i.e., increased expression of costimulatory molecule and reduced MR expression.

Effects of in vivo $1 \alpha$-D3 on ratio of cytokines IL-12/IL-10 level in MDDC cultures of GD patients

Our study is the first study that evaluates the effect of oral vitamin D supplementation on the ratio of inflammatory (IL-12) and antiinflammatory (IL-10) cytokine level in supernatant of MDDC culture of patients with autoimmune disease. Changes in IL-12/IL-10 ratio are strongly affected by IL-12 cytokine. Our study has not been able to show the effect of oral vitamin D supplementation on the ratio of IL-12/IL-10 levels in patients with GD since the ratio of IL-12/IL-10 levels among two groups was not significant.

Thyroid hormone (fT4) affects DC maturation; therefore, it correlates with IL-12 cytokines produced by DC [7]. The IL-12 cytokine level is high in GD patients who are still hyperthyroid and decreases with ATD treatment. This IL-12 decrement is correlated with decreased fT4 level $(r=0.5)$ [27]. In our study, we did not find any correlation between fT4 and IL-12 cytokine level ( $\mathrm{r}=-0.06)$. The absence of correlation between fT4 and IL-12 cytokine level in our study might be due to the relatively short monitoring period (8 weeks) compared to other studies. A short monitoring period caused IL-12 level was not optimally reduced; therefore, our study did not find any significant correlation between fT4 and IL-12 levels. A study that shows correlation between fT4 and IL-12 cytokine levels had 12 months of monitoring period, and it demonstrates continuous reduction of IL- 12 level until the $5^{\text {th }}$ month of treatment although the fT4 level has reached the normal limit on the $2^{\text {nd }}$ month of treatment [27].

Another clinical study associated with the role of vitamin D from sun exposure on changes of cytokines of GD patients was conducted in Jakarta with 32 GD patients, who were categorized into 2 groups, i.e., the exposure and non-exposure groups [28]. Results of the study showed that sun exposure three times a week for 20-30 minutes of each exposure for four weeks increased vitamin D level as much as $20 \%$ and inhibited the increment of IL-4 cytokine levels. IL- 4 cytokine is a major stimulant for DC for IL-12 secretion; therefore, it is assumed that lower IL-4 cytokine will reduce IL-12 cytokine and it may lead to reducing activation of T cells. The study of sun exposure describes the important role of cytokines in GD [29].

\section{Effects of oral 1 $\alpha$-D3 on fT4 level in GD patients}

A study by Kawakami-Tami et al. [20] demonstrates that $1 \alpha$-D3 supplementation decreases thyroid hormone (fT4, fT3) faster in hyperthyroid phase and increases TSH level faster in achieving euthyroid phase. After euthyroid, there is no difference of fT3 and fT4 levels between the $1 \alpha$-D3 and placebo groups. In our study, both the mean of fT4 level after supplementation and percentage of fT4 level reduction were not different between both groups. There was an interesting phenomenon on the fT4 changes, i.e., in the placebo group there were two patients who experienced increased fT4 level during the study; while there was no patients in the treatment group who experienced increased fT 4 level. It can be a good reason for conducting further studies on vitamin D supplementation in GD.

The response of fT4 level decrement in the first 8 weeks of our study was not as fast as the response found in Kawakami-Tani et al. study. In the Kawakami-Tani et al. study, on the $8^{\text {th }}$ week, the fT4 level reached normal level in both groups and the TSH level began to elevate; while in our study, 8 out of 12 GD patients in the treatment group and 6 out of $13 \mathrm{GD}$ patients in the placebo group still had fT4 level above normal limit and TSH levels had not increased among all patients. The difference in results between our study and Kawakami-Tani et al. study might be influenced by the ATD used; our study used PTU while Kawakami-Tani et al. study used MMI. The transition of hyperthyroid phase to euthyroid phase in our study has not been completed yet; therefore, immunological changes, particularly for DC maturation still cannot be demonstrated.

\section{Study limitation}

For a clinical study, the in vivo design of our study has some limitation regarding the number of patients and a relatively short period of supplementation (8 weeks) compared to the previous clinical study (24 weeks) [20]. The small number of patients with heterogeneous patient profiles caused difficulty to observe and to obtain changes in the immune response during the short period observation. 
In addition to those factors, the baseline vitamin $\mathrm{D}$ level found in the patients also affects the outcomes. The effect of vitamin D supplementation will be more noticeable in patients with low baseline vitamin D level (deficiency and insufficiency). In patients with vitamin D level that close to normal range, as found in our study, we need a longer observation period since the response to vitamin D supplementation tends to occur in sloping trends.

\section{CONCLUSION}

The effects of oral $1 \alpha$-D3 supplementation for 8 weeks on DC maturation of GD patients have not been clearly demonstrated in this study yet.

\section{ACKNOWLEDGMENTS}

This study was supported by DIKTI (The Directorate General of Higher Education) Research Grants 2014 and by Division of Endocrinology, Department of Medicine, Faculty of Medicine, Universitas Indonesia. The content is solely the responsibility of authors and does not necessarily represent general view of Universitas Indonesia.

\section{REFERENCES}

1. Tamura M, Matsuura B, Miyauchi S, Onji M. Dendritic cells produce interleukin-12 in hyperthyroid mice. Eur $\mathrm{J}$ Endocrinol 1999;141(6):625-9.

2. Banchereau J, Steinman RM. Dendritic cells and the control of immunity. Nature 1998;392(6673):245-52.

3. Liang S, Ristich V, Arase H, Dausset J, Carosella ED, Horuzsko A. Modulation of dendritic cell differentiation by HLA-G and ILT4 requires the IL6-STAT3 signaling pathway. Proc Natl Acad Sci U S A 2008;105(24):8357-62.

4. Inoue N, Watanabe M, Morita M, Tatusmi K, Hidaka Y, Akamizu T, et al. Association of functional polymorphisms in promoter regions of IL5, IL6 and IL13 genes with development and prognosis of autoimmune thyroid disease. Clin Exp Immunol 2011;163:318-23.

5. Khalilzadeh O, Anvari M, Esteghamati A, Momen-Heravi F, Mahmoudi M, Rashidi A, et al. The interleukin-1 family gene polymorphisms and graves disease. Ann Endocrinol 2010;71:281-5.

6. Croizet K, Rabilloud R, Kostrouch Z, Nicolas JF, Rousset B. Culture of dendritic cells from a nonlymphoid organ, the thyroid gland: Evidence for TNFa-dependent phenotypic changes of thyroid-derived dendritic cells. Lab Invest 2000;80(8):1215-25.

7. Dedecjus M, Stasiolek M, Brzezinski J, Selmaj K, Lewinski A. Thyroid hormone influence human dendritic cells phenotype, function and subset distribution. Thyroid 2011;21:533-40.

8. Hughes GC, Clark EA. Regulation of dendritic cells by female sex steroids: Relevance to immunity and autoimmunity. Autoimmunity 2007;40:470-81.

9. Duperrier K, Farre A, Bienvenu J, Bleyzac N, Bernaud J, Gebuhrer L, et al. Cyclosporin A inhibits dendritic cell maturation promoted by TNF- $\alpha$ or LPS but not by double stranded RNA or CD40L. J Leukoc Biol 2002;72:953-61.

10. Berer A, Stöck1 J, Majdic O, Wagner T, Kollars M, Lechner K, et al. 1,25-dihydroxyvitamin D3 inhibits dendritic cell differentiation and maturation in vitro. Exp Hematol 2000;28(5):575-83

11. Wahono CS, Rusmini H, Soelistyoningsih D, Hakim R, Handono K, Endharti AT, et al. Effects of $1,25(\mathrm{OH})_{2} \mathrm{D}_{3}$ in immune response regulation of systemic lupus erithematosus (SLE) patient with hypovitamin D. Int J Clin Exp Med 2014;7:22-31.
12. Bartels LE, Jøgersen SP, Bendix M, Hvas CL, Agnholt J, Agger R, et al. 25-hydroxy vitamin D3 modulates dendritic cell phenotype and function in Crohn's disease. Inflammopharmacology 2013;21(2):177-86.

13. Bartels LE, Bendix M, Hvas CL, Jørgensen SP, Agnholt J, Agger R, et al. Oral vitamin D3 supplementation reduces monocyte-derived dendritic cell maturation and cytokine production in Crohn's disease patients. Inflammopharmacology 2014;22:95-103.

14. Bartosik-Psujek H, Tabarkiewicz J, Pocinska K, Stelmasiak Z, Rolinski J. Immunomodulatory effects of vitamin $\mathrm{D}$ on monocyte-derived dendritic cell in multiple sclerosis. Mult Scler 2010;16(12):1513-6.

15. Abou-Raya A, Abou-Raya S, Helmii M. The effect of vitamin D supplementation on inflammatory and hemostatic markers and disease activity in patients with systemic lupus erythematosus: A randomized placebo-controlled trial. J Rheumatol 2013;40(3):265-72

16. Agmon-Levin N, Theodor E, Segal RM, Shoenfeld Y. Vitamin D in systemic and organ-specific autoimmune diseases. Clinic Rev Allerg Immunol 2013;45(2):256-66.

17. Pani MA, Regulla K, Segni M, Hofmann S, Hüfner M, Pasquino AM, et al. A polymorphism within the vitamin D-binding protein gene is associated with Graves' disease but not with Hashimoto's thyroiditis. J Clin Endocrinol Metab 2002;87(6):2564-7.

18. Zhou $\mathrm{H}, \mathrm{Xu} \mathrm{C}, \mathrm{Gu} \mathrm{M}$. Vitamin D receptor (VDR) gene polymorphisms and Graves' disease: A meta-analysis. Clin Endocrinol (Oxf) 2009;70(6):938-45.

19. Yasuda T, Okamoto Y, Hamada N, Miyashita K, Takahara M, Sakamoto F, et al. Serum vitamin D levels are decreased in patients without remission of Graves' disease. Endocrine 2013;43(1):230-2.

20. Kawakami-Tani T, Fukawa E, Tanaka H, Abe Y, Makino I. Effect of 1a-hydroxyvitamin D3 on serum levels of thyroid hormones in hyperthyroid patients with untreated Graves' disease. Metabolism 1997;46(10):1184-8.

21. Purnamasari D, Soewondo P, Djauzi S, Setiati S, Harahap A, Prihartono J. Effects of in vitro 1,25 dihydroxyvitamin d on maturation of dendritic cells in Graves' disease patients. Asian J Pharm Clin Res 2016;9(5):221-4

22. Budiyati AD, Setiyono A, Tarigan E, Wibowo H. The effect of alpha fetoprotein on NF-kB translocation in lipopolysaccharide induced monocyte-derived dendritic cell. Med J Indones 2012;21(2):97-101.

23. Hewison M. Vitamin D and the immune system: New perspectives on an old theme. Endocrinol Metab Clin North Am 2010;39(2):365-79.

24. Handono K, Gani AA, Ekawati M, Wahono S. Serum level of vitamin D and autoantibodies level in systemic lupus erythematosus (SLE) patients. J Pharm Biol Sci 2012;3:16-20.

25. Antico A, Tampoia M, Tozzoli R, Bizzaro N. Can supplementation with vitamin $\mathrm{D}$ reduce the risk or modify the course of autoimmune disease? A systematic review of the literature. Autoimmun Rev 2012;12(2):127-36.

26. Bartels LE, Bendix M, Hvas CL, Jørgensen SP, Agnholt J, Agger R, et al. Oral vitamin D3 supplementation reduces monocyte-derived dendritic cell maturation and cytokine production in Crohn's disease patients. Inflammopharmacology 2013;22(2):95-103

27. Tamaru M, Matsuura B, Onji M. Increased levels of serum interleukin-12 in Graves' disease. Eur J Endocrinol 1999;141:111-6.

28. Purnamasari D, Soewondo P, Djauzi S. Sunlight-derived vitamin D affects interleukin-4 level, T helper 2 serum cytokines, in patients with Graves' disease: A prospective cohort study. Med J Indones $2015 ; 24: 228-33$.

29. Hunt PJ, Marshall SE, Weetman AP, Bell JI, Wass JAH, Welsh KI. Cytokine gene polymorphisms in autoimmune thyroid disease. J Clin Endocrinol Metab 2000;85(5):1984-8. 